\title{
Review of: "Compounds enhancing human sperm motility identified using a high-throughput phenotypic screening platform"
}

\author{
Yue Liư \\ 1 Shanghai Second Medical University
}

Potential competing interests: The author(s) declared that no potential competing interests exist.

The authors of this manuscript describe the results of a high-throughput screening platform investigating the role of compounds in improving sperm motility. They show convincingly that a series of small molecules including phosphodiesterase inhibitors are identified to increase sperm motility, with tiny tracedoes and rapid response. Overall this is an interesting study, however there are a few concerns:

1) The data of the sperm motility were detected by HTS system at least 3 replicates (once in initial screening and twice in dose response experiments). In Figure2C and Table3, are the replicates calculated or statistical analysis performed?

2) The authors should explain why choose curvilinear velocity (VCL) to represent sperm motility, not VAP or VSL? The exact meaning of VCL is unfamiliar perhaps to many readers. As reported by Gruber and colleagues (Gruber 148 et al., 2020), HTS system is able to quantify all these kinetic parameters of human spermatozoa.

3) In Figure2B, tracks of spermatozoa are too small to distinguish. An enlarged image for sperm track may be helpful in explaining this approach.

4) Although such quick increase of sperm motility by the compounds is an exciting discovery, the potential harmful impacts on sperm function, such as accelerated acrosome reaction, induced excessive ROS and DNA damage, need further consideration. 